\title{
Study on Three-Dimensional Inner Flow Field Characteristics and Performance of Scramjet
}

\author{
Feng Gao*, Jie Zhao, Cheng Tao Zhang \\ Air and Missile Defense College, Air Force Engineering University, 710051 Shaanxi Xian, China
}

\begin{abstract}
The three-dimensional characteristics and performance of the flow field in the inlet of the scramjet engine were numerically simulated by CFD software. The flow characteristics in the width direction of the inlet and the influence of the aspect ratio on the performance of the inlet were studied. The calculation results show that the inlet flow has obvious three-dimensional characteristics, and the flow field structure is different in the width direction from the middle symmetrical section to the side wall surface, the Mach number is smaller and smaller, the static pressure is lower and lower, and the static temperature is higher, the greater the total pressure. The aspect ratio has little effect on the Mach number and static temperature of the outlet section of the inlet, but it has a great influence on the static pressure and total pressure. Within a reasonable range, the aspect ratio is doubled, the static pressure is increased by about $40 \%$, and the total pressure is increased by about $84 \%$. The inlet flow coefficient and the total pressure recovery coefficient increase as the aspect ratio increases. Within a reasonable range, the aspect ratio is doubled, the inlet flow coefficient is increased by approximately $53 \%$, and the total pressure recovery coefficient is increased by approximately $83 \%$.
\end{abstract}

\section{INTRODUCTION}

Hypersonic vehicles have a wide range of applications in the military and civilian fields in the future, and research institutes at home and abroad have actively carried out research and have made remarkable achievements. The scramjet engine is the core of the hypersonic vehicle (Fig. 1). Researchers have carried out a large number of experiments and numerical simulations on the structure optimization, performance evaluation, flow and combustion calculation of the scramjet engine and its inlet. Effective work [1, 2, 3, 4]. The flow field inside and outside the hypersonic inlet is very complicated and is a typical three-dimensional flow. The flow characteristics in the width direction have a great influence on the performance of the inlet. Therefore, the aspect ratio of the inlet becomes the important parameter that affects the whole inlet and even the whole scramjet, which must be paid great $[5,6]$. Generally speaking, forebody compression of scramjet is composed of three to four Wave hierarchies. However, recent researches reflect that it may influence the characteristics of airinlet inner flow field when importing side-wall compression in the compacting board of air-inlet. So, the numerical simulation on characteristics and performances of the three-dimension scramjet air-inlet flow field was carried out in this paper, the detailed characteristics of inlets' inner flow field and the influence of the aspect-ratio on air-inlet performance were simply studied.

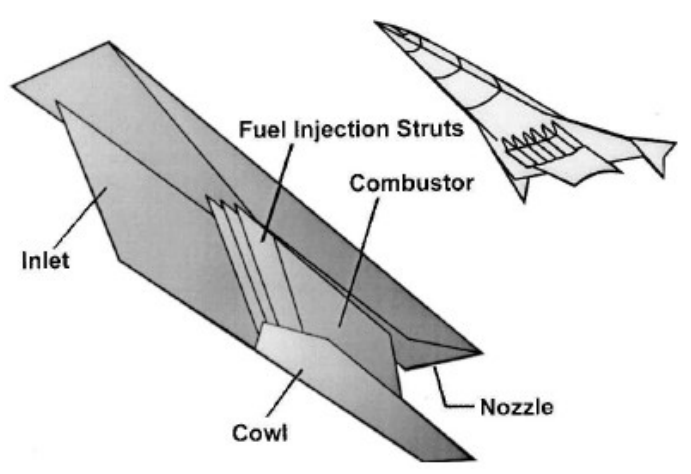

Fig.1 airframe-integrated engine configuration.

\section{COMPUTATIONAL METHODOLOGY}

\subsection{Air-inlet geometry model and Parameters}

The computational model adopted in the present study uses the three dimension inlet model in paper[7], the geometry structures and parameters as shown in the Fig. 2 and Table 1.The aspect-ratio of the air-inlet are 1, 3, 4,

\footnotetext{
* Corresponding author: gnning@sina.com
} 
7, besides, side-wall compression is necessary. The nominal Mach 6 inlet flow entry stagnation conditions were $P_{\mathrm{t}}=1718.6 \mathrm{~Pa}$ and $T_{\mathrm{t}}=4137551.21 \mathrm{~K}$.

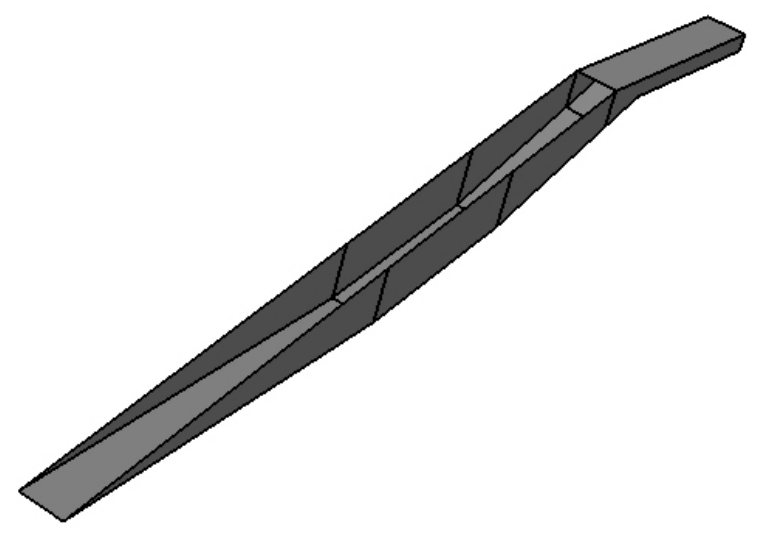

Fig.2 the three dimension inlet model

Table 1. mass average parameters of inlet' exit

\begin{tabular}{|c|c|c|c|c|c|}
\hline \multicolumn{2}{|c|}{ Average parameters } & Ma & $\begin{array}{c}\text { Static } \\
\text { temperature (K) }\end{array}$ & $\begin{array}{c}\text { Static pressure } \\
(\mathrm{Pa})\end{array}$ & $\begin{array}{c}\text { Total } \\
\text { pressure(Pa) }\end{array}$ \\
\hline \multirow{3}{*}{ Computational model } & & 2.27 & 951.924 & 129296.7 & 1734163 \\
\hline \multirow{2}{*}{ 3D } & $r=3$ & 2.02 & 1069.5 & 54988.34 & 492510 \\
\cline { 2 - 6 } & $r=5$ & 2.18 & 1000.2 & 69432.35 & 821167 \\
\cline { 2 - 6 } & $r=7$ & 2.19 & 1018.2 & 84151.72 & 996180 \\
\hline
\end{tabular}

\subsection{Computational method of air-inlet parameters}

The air-inlet parameters are composed of total pressure recovering coefficient, flow rate coefficient, additional resistance coefficient and ram ratio. This paper mainly uses total pressure recovering coefficient and flow rate coefficient to evaluate the performance of the scramjet air-inlet.

(1) Total pressure recovering coefficient is defined as ratio between inlet average total pressure and outlet average total pressure which is to assess the airflow kinetic energy loss in the process of stagnation. Equation for this is given by Eq. (1)

$$
\sigma=\frac{P_{t e}}{P_{t 0}}
$$

(2) Flow rate coefficient ratio, ratio between actual inlet flow rate coefficient and inlet free flow rate coefficient without being disturbance. Equation for this is given by Eq. (2)

$$
\varphi=\frac{A_{0}}{A_{c}}
$$

\subsection{Computational Methodology}

In addition to the compressible Navier-Stokes and energy equations, the K-epsilon Turbulence model is utilized in this paper. In near wall region nonequilibrium wall function method is adopted. In order to catch the shock wave, double-precision equation solver and implicit coupling method are applied. Second Order Upwind was selected in discretization scheme. Ideal-gas model is used for density for all fluid entering the chamber. All fluid exit planes are designated as pressureoutlets and all fluid entrance planes are designated as pressure-inlets while all remaining faces were designated as wall boundaries.

\subsection{Initial conditions and Convergence criteria}

First, initialize the flow field with the calculation result of steady-state. The shock wave increases residuals, so when every parameter's residual is below $10^{-3}$ and keeping steady, criterion for convergence is met.

\section{RESULTS AND DISCUSSION}

\subsection{Characteristics of air-inlet flow field in the width direction}

Fig.3 shows the inlet static temperature contours at different width. It is seen that the first wave of external compression wave decrease from the symmetry plane to side-wall, this results in the deformation of the first shock wave. The external compression wave does not come across at cowl lip. During compression segment, shock wave intensity decrease from the symmetry plane to side-wall. The main reason is the side-wall also has 
boundary layer. Along with the boundary layer becoming thicker, side-wall compression effect interferes with inclined plane compression effect. This may result in decline of flow rate performance, total pressure recovering performance and comprehensive performance. As a result, the plan cannot meet the requirement of the scramjet.

Fig. 4 to Fig.7 show the inlet exit' Mach number, static temperature, static pressure and total pressure

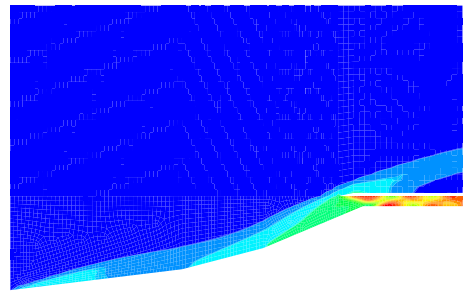

$\mathrm{Z}=0$

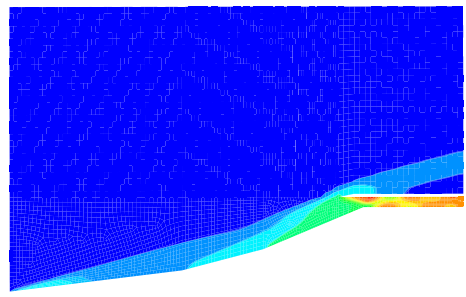

$\mathrm{Z}=50$

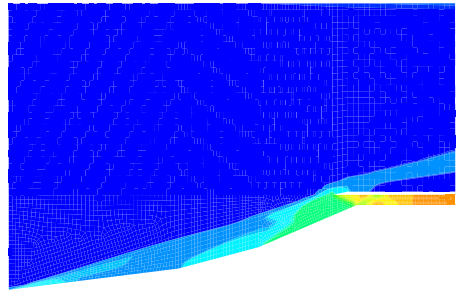

$\mathrm{Z}=90$

Fig.3 The inlet static temperature contours at different width

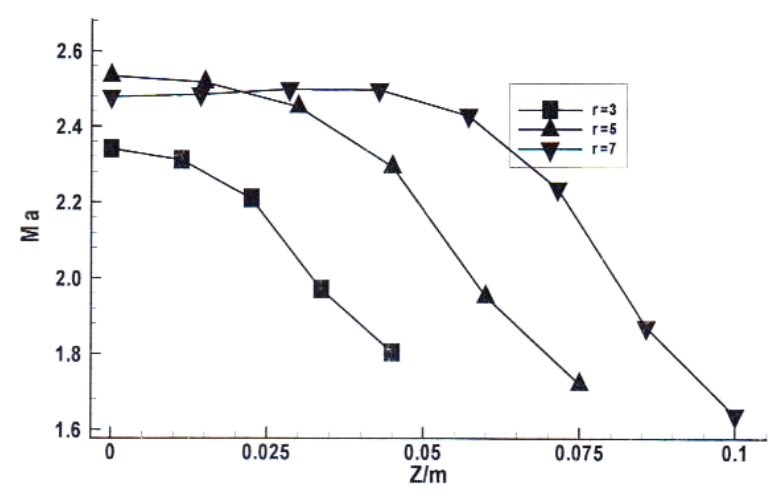

Fig.4 The inlet exit' Mach number distributional curve along width direction

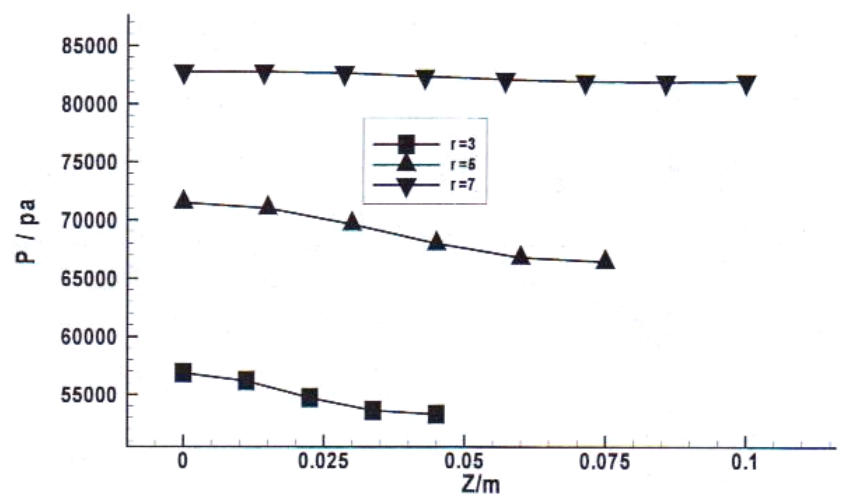

Fig. 6 The inlet exit' static pressure distributional curve along width direction distributional curves along width direction. It is seen that from symmetry plane to side-wall, the Mach number, total pressure and static temperature decrease a bit little, but static temperature increases. Because of the influence of wall, the flow conditions are different, there are obvious three-dimensional phenomena and influences in inlet flow.

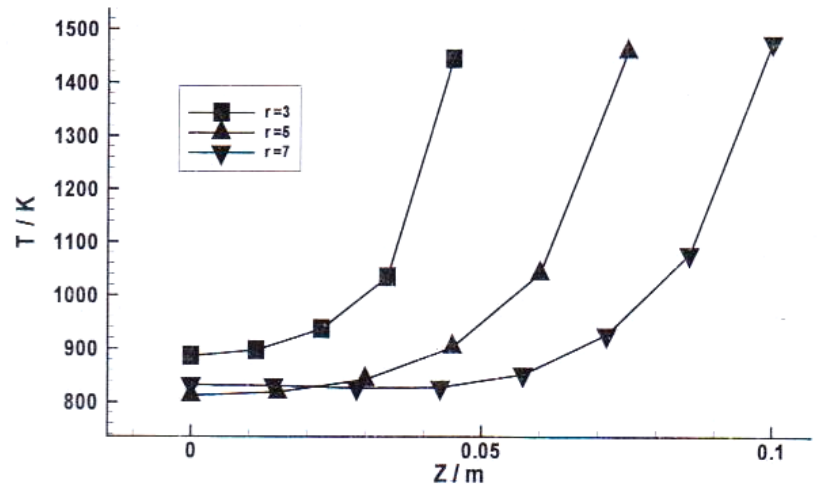

Fig. 5 The inlet exit' static temperature distributional curve along width direction

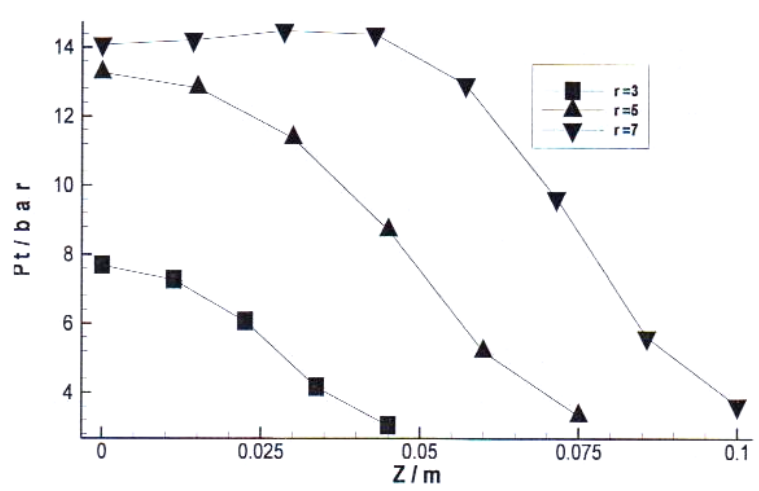

Fig. 7 The inlet exit' total pressure distributional curve along width direction

3.2 The influence to air-inlet in different aspectratio 
Fig.8 shows the different aspect-ratio versus the Mach number, static temperature and static pressure at the symmetry plane. It is seen the aspect-ratio has great effects on the static pressure and total pressure but has

Fig.9 shows the inlet flow coefficient and the total pressure recovery coefficient versus the aspect-ratio. It is seen that the nature increase in the air-inlet flow rate coefficient and total pressure recovering coefficient as the aspect-ratio is increased. Within a reasonable range, the aspect ratio is doubled, the inlet flow coefficient is increased by approximately $53 \%$, and the total pressure recovery coefficient is increased by approximately $83 \%$.The main reason is the ratio of the complex ThreeDimensional Flow Field versus the whole flow field is different, so the performances of different aspect-ratio air-inlets are different. If the aspect-ratio reduces the ratio of the complex Three-Dimensional Flow Field versus the whole flow field is increased, the ThreeDimensional Flow' interferences to the whole flow field become obvious, this increases the total pressure loss. The influence of Three-Dimensional Flow makes the little effect on the Mach number and static temperature. Within a reasonable range, the aspect ratio is doubled, the static pressure is increased by about $40 \%$, and the total pressure is increased by about $84 \%$.

whole flow field more nouniform, the overflow increases, so the flow rate coefficient decreases.

At the same time, with the increase of aspect-ratio the ratio of the complex Three-Dimensional Flow Field versus the whole flow field decreases, the ThreeDimensional Flow has fewer influences to the performance of air-inlet. There is little improvement to air-inlet performance when increasing aspect-ratio.

Comparing mass average parameters of inlet' exit in Table 1, the Mach number of outlet is a bit high but the temperature of outlet is a bit low. The Two- Dimensional computational result is higher than the ThreeDimensional computational result. Wall-effect may lead to this. So, it is necessary to take the influence of Walleffect into consideration when designing air-inlet in major engineering design projects.
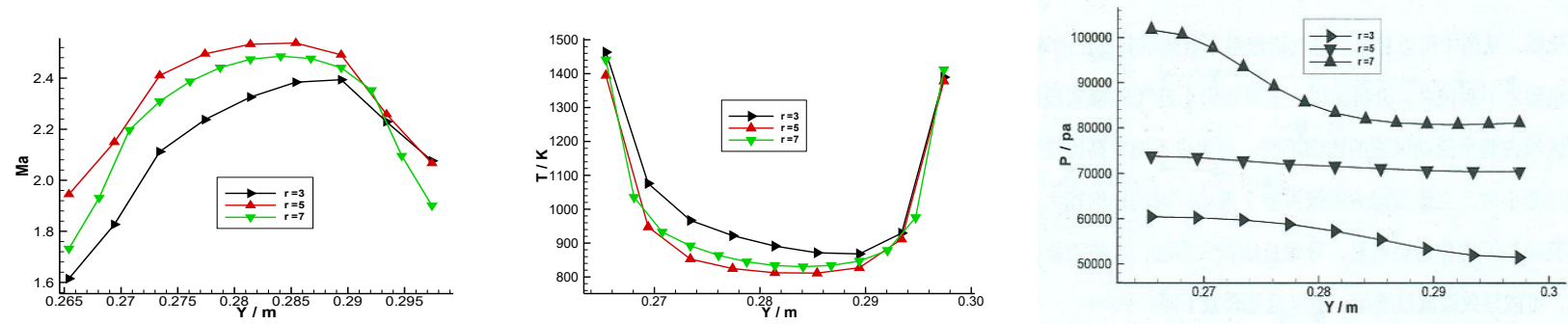

Fig. 8 The Mach number and the static and total temperature distributional curve in the aspect-ratio
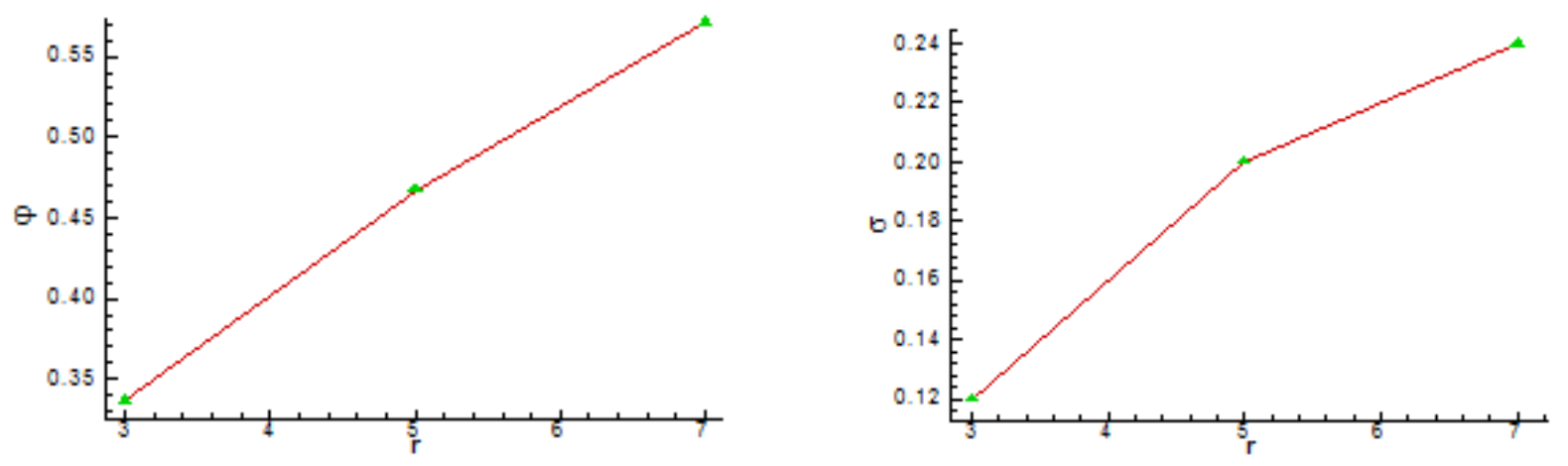

Fig. 9 The inlet flow coefficient and the total pressure recovery coefficient vary with the aspect-ratio

\section{Summary and Conclusions}

In this paper, numerical simulation on scramjet inner flow field was carried out. The influence of the ThreeDimensional effect on the flow and performance of the scramjet inlet were studied, conclusions are as follows:

(1) From symmetry plane to side-wall, the Mach number, total pressure and static temperature decrease a bit little, but static temperature increases. The flow conditions are different, there are obvious threedimensional phenomena and influences in inlet flow.
(2) The aspect-ratio has great effects on the static pressure and total pressure but has little effect on the Mach number and static temperature. Within a reasonable range, the aspect ratio is doubled, the static pressure is increased by about $40 \%$, and the total pressure is increased by about $84 \%$.

(3) The nature increase in the air-inlet flow rate coefficient and total pressure recovering coefficient as the aspect-ratio is increased. Within a reasonable range, the aspect ratio is doubled, the inlet flow coefficient is increased by approximately $53 \%$, and the total pressure recovery coefficient is increased by approximately $83 \%$. 


\section{References}

1. Ajay Kumar, Journal of Aircraft, 19, 7 (1982)

2. Andreas K. Flock, Ali Gülhan, Journal of Propulsion and Power, 33, 6 (2017)

3. Andreas K. Flock, Ali Gülhan, AIAA Journal, 53, 9 (2015)

4. Robert J. Yentsch;Datta V. Gaitonde, Journal of Propulsion and Power, 31, 1 (2015)

5. Jinhu Feng, Feng Gao, Journal of Air Force Engineering University(Natural Science Edition), 10, 4(2009)

6. Feng Gao, Hongyu Wang, Winged Missiles Journal, 1: $80 \sim 84(2014)$

7. Zhilin He, Feng Gao, Winged Missiles Journal, 4: $82 \sim 87(2010)$ 